\title{
Mini review study of acetaminophen overdose poisoning and associated factors in cases from Poison
}

\author{
Al-Farga Ammar*1, Mohammed Alkhuzaee ${ }^{1}$, Naji Alsulami ${ }^{1}$, Anas Bushah ${ }^{1}$, Ali Almalki ${ }^{1}$, \\ Abdulhakim Torkistani ${ }^{1}$, Mohammed Baeshen ${ }^{2}$, Mohamed Afifi ${ }^{1}$ \\ 1 Department of Biochemistry, Faculty of Science, University of Jeddah, Jeddah, Saudi Arabia \\ 2 Department of Biology, Faculty of Science, University of Jeddah, Jeddah, Saudi Arabia \\ *Corresponding Author: Ammar Alfergah, Department of Biochemistry, Faculty of Science, University of Jeddah, Jeddah, Saudi \\ Arabia, Email: alfergah83@gmail.com
}

Received: February 03, 2019; Published: March 28, 2019

\section{Abstract}

Acetaminophen (APAP) poisoning is among the most common causes of medication-related poisoning and death. Acetaminophen poisoning may occur following a single acute ingestion or through the repeated ingestion of supratherapeutic amounts. The management of the acetaminophen-poisoned patient may include stabilization, decontamination, and administration of $\mathrm{N}$-acetylcysteine, a specific antidote. The duration of $\mathrm{N}$-acetylcysteine treatment is determined by the type of ingestion and the presence or absence of elevated serum alanine aminotransferase (ALT) concentrations. Acetaminophen toxicity is one of the most common causes of both intentional and unintentional poisoning in the whole world. Acetaminophen is often recommended by doctors and highly utilized by patients in both prescription and OTC products for a variety of conditions. The FDA has long been updating its recommendations regarding acetaminophen use to help improve patient safety. Acetaminophen is often recommended by doctors and highly utilized by patients in both prescription and OTC products for a variety of conditions, making it undoubtedly one of the most common drugs encountered by pharmacists. This article reviews the etiology, signs, and symptoms of acetaminophen toxicity and associated factors in cases from Poison.

Keywords: Mini review, Acetaminophen, Poisoning.

\section{INTRODUCTION}

Paracetamol, a first-line therapy for mild-to-moderate pain due to its efficacy, tolerability, and affordability. Acetaminophen (Paracetamol) is utilized worldwide for its pain relieving and antipyretic properties. It is broadly accessible and present in numerous medical and non-remedy prescription. Paracetamol (acetaminophen) is a mellow pain relieving and antipyretic specialist which is ordinarily utilized around the world [1]. In remedial dosages (for grown-ups $500 \mathrm{mg}$ to $1000 \mathrm{mg}$, three or four times each day), paracetamol has a couple of unfavorable occasions. Paracetamol is ordinarily taken in overdose either unintentionally or purposefully [2], and in numerous nations, it is the most widely recognized single compound taken in overdose [3]. In general, a single dose of more than $10 \mathrm{~g}$ or $150 \mathrm{mg} / \mathrm{kg}$ to $200 \mathrm{mg} / \mathrm{kg}$ of paracetamol conveys a risk of liver damage, yet little portions may also cause liver harm [4], especially in individuals with constant liquor misuse or anorexia. Paracetamol lethality is the main source of intense liver disappointment in some high-salary nations [5-7]. One huge forthcoming observational companion investigation of 31 liver infection and transplant focuses in the US, enlisting 2070 members with intense liver disappointment somewhere in the range of 1998 and 2013, found that paracetamol harming was the reason in half of the members [8]. It was not until the1970s that several antidotes that recharge glutathione and detoxify N-acetyl-pbenzoquinone imine (NAPQI) were produced; this included methionine, cysteine, cysteamine, and dimercaprol. Oral methionine and intravenous acetylcysteine have been utilized as counteractants in the UK from this time onwards [9]. In one observational investigation from Edinburgh, intravenous acetylcysteine first-line was professed to be similarly as powerful as cysteamine and methionine and free of unfavorable impacts. From that point onward, acetylcysteine has been acknowledged as a counteractant for paracetamol overdose either intravenously or orally. A great part of the proof for its utilization and efficacy comes from observational thinks about. Acetylcysteine has now turn into the backbone and standard 
treatment for paracetamol harming and can either be directed as a 20-to 21-hour intravenous acetylcysteine routine or an oral acetylcysteine routine [10-12]. Acetaminophen ( $\mathrm{N}$-acetyl-paraaminophenol, paracetamol, APAP) toxicity is common especially due to the fact the remedy is so quite simply reachable and there is an appreciation that it is very safe. More than 60 million consume acetaminophen on a weekly basis [13]. Acetaminophen is used in many products in aggregate with other preparations, especially with opioids and diphenhydramine. Many people are not conscious that it is contained in these mixture medicinal drugs [14]. Acetaminophen (APAP) is one of the most often used oral analgesics and antipyretics. It has an excellent security profile when administered in suited therapeutic doses. Acetaminophen poisoning is among the most frequent reasons of medicationrelated poisoning and death. Acetaminophen poisoning might also manifest following a single acute ingestion or thru the repeated ingestion of supratherapeutic amounts. The acetaminophen products are extensively used as OTC drug so the opportunity of toxicity by means of overdosing of this drug is high.

\section{Acetaminophen Toxicity Symptoms and Treatment}

Acetaminophen toxicity or overdose can occur purposefully (when a person knowingly takes more than the recommended maximum daily dose) or accidentally (when a person is unaware, they are taking multiple products containing acetaminophen and exceeds the recommended maximum daily dose). The maximum recommended acetaminophen dosage is 4 grams/day in an adult and $90 \mathrm{mg} / \mathrm{kg} / \mathrm{day}$ in children [14]. People with underlying liver disease or those with chronic alcohol consumption are at an increased risk of developing hepatotoxicity (liver damage from chemicals) with use of acetaminophen.

\section{Acetaminophen Toxicity Diagnosis}

A doctor's first step in diagnosing acetaminophen toxicity is to get a complete history, including the time the medication was ingested, the amount of medication that was ingested, and what form of the medication was ingested. A diagnosis of acetaminophen toxicity is usually confirmed through diagnostic tests, including an acetaminophen level, electrolytes, kidney function tests, amylase, lipase, liver function tests, complete blood count, and coagulation factors. Imaging studies, such as an ultrasound may be used to assess liver enlargement. A liver biopsy may also be ordered [15].

\section{Acetaminophen Toxicity Treatment}

Timing is a vital factor in the treatment of acetaminophen toxicity, and therefore doctors attempt to begin treatment of acetaminophen overdose within eight hours of ingestion in order to achieve the best possible outcome for the patient. The majority of patients survive acetaminophen toxicity with supportive care such as intravenous fluids and anti-nausea medication, activated charcoal, if used within one hour after ingestion, and antidotal therapy, including $\mathrm{N}$-acetylcysteine [13]. For patients who fail the above therapies and develop liver failure, liver transplantation may be the only treatment option. Doctors will determine if transplantation is necessary if the above tests are significantly abnormal and the patient has developed hepatic encephalopathy, a disorder of the brain caused by a dysfunctional liver.

\section{Clinical Presentation of paracetamol toxicity}

The clinical signs, as a rule, don't wind up clear for the initial 2448 hours after an intense overdose of paracetamol. Liver disappointment may happen between 2-7 days following the ingestion. The clinical course of paracetamol harmfulness is commonly separated into 4 stages. Stage 1 (0-24 hrs) The patient is generally asymptomatic or may give highlights like anorexia, sickness, regurgitating and disquietude. The Liver Function Tests demonstrates a mellow increment in the serum transaminase level (starts to rise around 12 hours after intense ingestion). Stage 2 (18-72 hrs) The patient for the most part encounter sickness, retching, stomach torment (right upper quadrant). On examination, delicacy is available on the correct upper quadrant; tachycardia and hypotension are generally present. Serum transaminase level keeps on rising. Stage 3 (72$96 \mathrm{hrs})$ Hepatic stage This is the most basic stage. The patient is seriously sick. Jaundice, coagulopathy with draining inclinations, hypoglycemia, hepatic fold, and hepatic encephalopathy happen because of hepatic rot and brokenness. Metabolic acidosis with intense renal disappointment (due to hepatorenal disorder) may create. Demise typically happens as an outcome of multi-organ disappointment. Stage (4 days-3 weeks) Recovery stage Patients who endure the basic ailment of stage 3 , are bound to enhance with goals of the indications and organ disappointment.

\section{Investigation}

The examinations incorporate the coordinated serum paracetamol fixation, liver capacity tests (counting prothrombin time or universal standardized proportion) and kidney work tests. These tests are expected to survey hazard and screen advance. The plasma centralization of paracetamol has prescient esteem, on the off chance that it lies over a semi-logarithmic chart which is acquired by joining the focuses between $1.32 \mathrm{mmol} / \mathrm{L}$ at 4 hours after ingestion to $0.33 \mathrm{mmol} / \mathrm{L}$ at 12 hours, at that point forecast is poor and genuine hepatic harm is probably going to happen.

\section{Pharmacology and Toxicity}

acetaminophen is rapidly absorbed from the gastrointestinal (GI) tract with peak concentrations

achieved within 90 minutes of a therapeutic dose. The presence of food in the stomach may delay the peak but not the extent of absorption [16]. Distribution is rapid with a volume of distribution $(\mathrm{Vd})$ of about $0.9 \mathrm{~L} / \mathrm{kg}$ and minimal protein binding at therapeutic concentrations [17]. The half-life of APAP is 2.0 to 2.5 hours. With hepatic injury, the half-life is prolonged to more than 4 hours [17]. Acetaminophen undergoes extensive hepatic metabolism. Approximately $85 \%$ of a therapeutic dose undergoes phase II conjugation to sulfated and glucuronidases metabolites that are renally eliminated. Of these two pathways, glucuronidation is predominant in adults, whereas sulfation predominates in children up to about 12 years of age []18. Up to $10 \%$ of APAP undergoes phase I oxidation to a reactive intermediate, N-acetyl-para-benzoquinone imine (NAPQI), which is normally conjugated with glutathione to nontoxic cysteine and mercapturate metabolites [19]. Cytochrome 2E1 is the primary cytochrome $\mathrm{p} 450$ (CYP) enzyme responsible for this oxidation. At supratherapeutic doses of APAP ( $>4 \mathrm{~g})$, sulfation becomes saturated with proportional increases in both glucuronidations and, more significantly, oxidation to NAPQI [19]. Smaller proportions of APAP are eliminated unchanged in the urine and by ring oxidation to a catechol derivative (Fig. 1) [19]. 


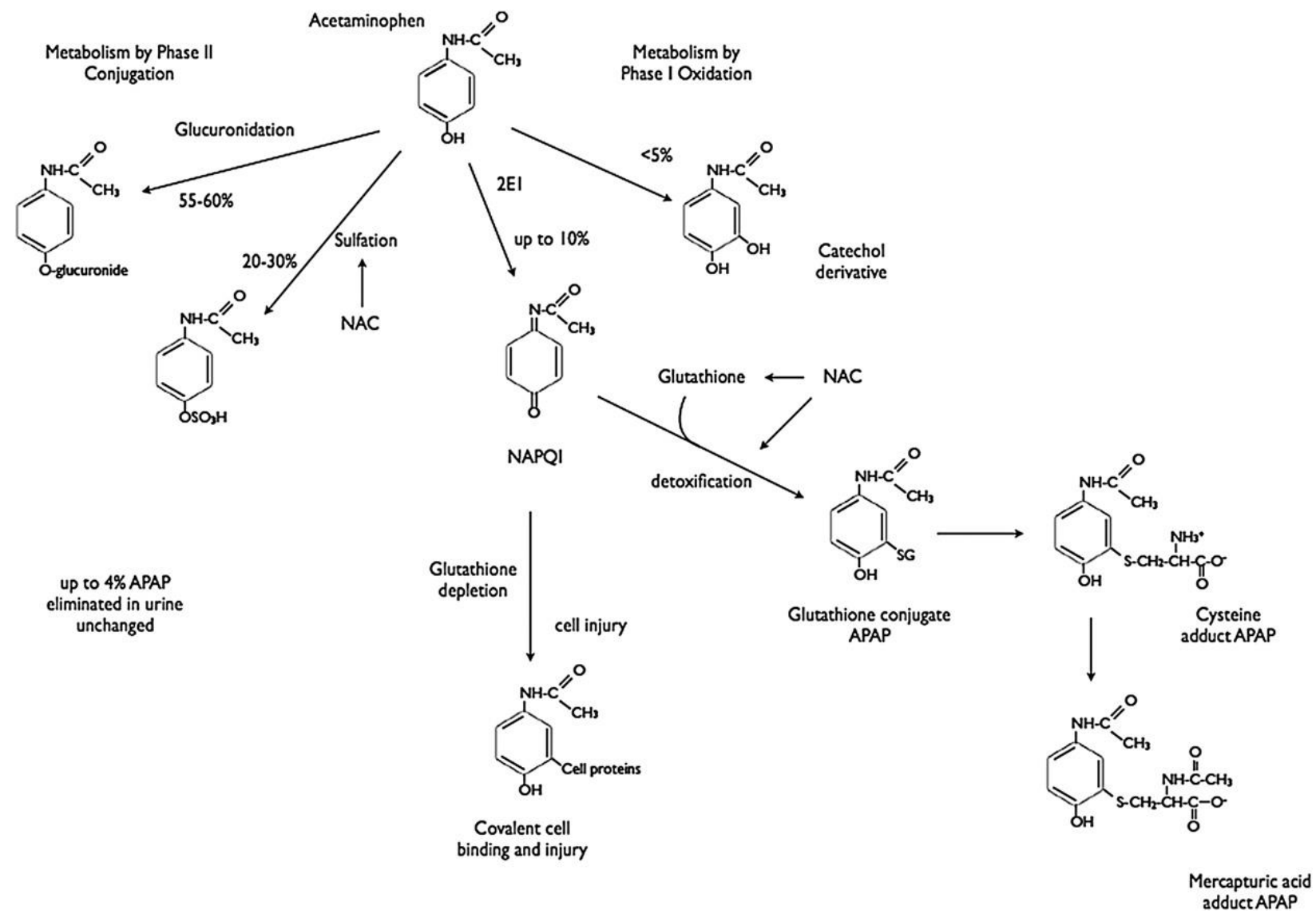

Fig 1: Metabolism of APAP. NAC, N-acetylcysteine

\section{Acknowledgements}

The authors would like to acknowledge the Priority Academic Program Development of Jiangsu, Higher Education Institutions, Wuxi city, Jiangsu province, People's Republic of China. We are also grateful to all the staff and students of the Research Centre of Functional Ingredients and Healthy Foods laboratory, and who helped us to bring the raw materials to China.

\section{Conflict of Interest}

None declared

\section{Financial Support}

None declared

\section{REFERENCES}

1. O'Grady JG. Paracetamol-induced acute liver failure. Journal of Hepatology 1997;26 (Suppl):41-6.

2. Buckley N, Eddleston M. Paracetamol (acetaminophen) poisoning. BMJ Clinical Evidence 2007;2007:2101.

3. Prescott LF (1980) Kinetics and metabolism of paracetamol and phenacetin. Br J Clin Pharmacol 10(Suppl 2):291S-298S.

4. Kwan D, Bartle WR, Walker SE. Abnormal serum transaminases following therapeutic doses of acetaminophen in the absence of known risk factors. Digestive Diseases and Sciences 1995;40(9):1951-5.

5. Lee WM. Acetaminophen and the U.S. Acute Liver Failure Study
Group: lowering the risks of hepatic failure. Hepatology (Baltimore, Md.) 2004;40(1):6-9. MEDLINE: PMID: 15239078.

6. Morgan O, Griffiths C, Majeed A. Impact of paracetamol pack size restrictions on poisoning from paracetamol in England and Wales: an observational study. Journal of Public Health 2005;27(1):1924. MEDLINE: PMID: 15637104.

7. Bernal W, Hyyrylainen A, Gera A, Audimoolam VK, McPhail MJW, Auzinger $\mathrm{G}$, et al. Lessons from look-back in acute liver failure? A single centre experience of 3300 patients. Journal of Hepatology 2013;59:74-80.

8. Reuben A, Tillman H, Fontana RJ, Davern T, McGuire B, Stravitz RT, et al. Outcomes in adults with acute liver failure between 1998 and 2013: an observational cohort study. Annals of Internal Medicine 2016;164(11):724-32.

9. McElhatton PR, Sullivan FM, Volans GN. Paracetamol overdose in pregnancy analysis of the outcomes of 300 cases referred to the Teratology Information Service. Reproductive Toxicology (Elmsford, N.Y.) 1997;11(1):85-94.

10. Smilkstein MJ, Bronstein AC, Linden C, Augenstein WL, Kulig KW, Rumack BH. Acetaminophen overdose: a 48hour intravenous Nacetylcysteine treatment protocol. Annals of Emergency Medicine 1991;20(10):1058-63.

11. Woo OF, Mueller PD, Olson KR, Anderson IB, Kim SY. Shorter duration of oral $\mathrm{N}$-acetylcysteine therapy for acute acetaminophen overdose. Annals of Emergency Medicine 2000;35(4):363-8.

12. Williamson K, Wahl MS, Mycyk MB. Direct comparison of 20-hour IV, 36-hour oral, and 72-hour oral acetylcysteine for treatment of acute acetaminophen poisoning. American Journal of Therapeutics 2013;20(1):37-40.

13. Athersuch TJ, Antoine DJ, Boobis AR, Coen M, Daly AK, Possamai L, Nicholson JK, Wilson ID. Paracetamol metabolism, 
hepatotoxicity, biomarkers and therapeutic interventions: a perspective. Toxicol Res (Camb). 2018 May 08;7(3):347-357.

14. Jasani B, Weisz DE, McNamara PJ. Evidence-based use of acetaminophen for hemodynamically significant ductus arteriosus in preterm infants. Semin. Perinatol. 2018 Jun;42(4):243-252.

15. Bunchorntavakul C, Reddy KR. Acetaminophen-related Hepatotoxicity. Clin Liver Dis.2013;17:587-607. doi: 10.1016/j.cld.2013.07.005.

16. Anon, editor. Tylenol professional product information. McNeil Consumer Healthcare, Division of McNEIL-PPC, Inc, Fort Washington, PA, 19034, USA; 2010.

17. Forrest JA, Clements JA, Prescott LF. Clinical pharmacokinetics of paracetamol. Clin Pharmacokinet 1982;7(2):93-107.

18. Prescott LF. Kinetics and metabolism of paracetamol and phenacetin. Br J Clin Pharmacol 1980;10(Suppl 2):291S-8S.

19. Gelotte CK, Auiler JF, Lynch JM, et al. Disposition of acetaminophen at 4,6 , and $8 \mathrm{~g} /$ day for 3 days in healthy young adults. Clin Pharmacol Ther 2007;81(6):840-8. 